\title{
LEAF PERFORMANCES OF CASSAVA GENOTYPES IN DIFFERENT SEASONS AND ITS RELATIONSHIP WITH BIOMASS
}

\author{
Warunyu PHOSAENGSRI ${ }^{1}$, Poramate BANTERNG ${ }^{1,2^{*}}$, Nimitr VORASOOT ${ }^{l}$, \\ Sanun JOGLOY ${ }^{1,2}$, Piyada THEERAKULPISUT ${ }^{3}$ \\ ${ }^{1}$ Khon Kaen University, Faculty of Agriculture, Khon Kaen, THAILAND \\ ${ }^{2}$ Khon Kaen University, Faculty of Agriculture, Plant Breeding Research Center for Sustainable \\ Agriculture, Khon Kaen, THAILAND \\ ${ }^{3}$ Khon Kaen University, Faculty of Science, Khon Kaen, THAILAND \\ *Corresponding author: bporam@kku.ac.th
}

Received: 20.06.2018

\begin{abstract}
Information on leaf performances in different growing seasons of cassava could help achieve the recommendation regarding suitable cassava genotypes for particular planting date and a new plant type to improve cassava productivity. The objective of this study was to investigate leaf growth habit and biomass of the four cassava genotypes grown under irrigated condition in different growing seasons. The four cassava genotypes, i.e., Kasetsart 50, Rayong 9, Rayong 11 and CMR38-125-77 were grown at 30 June 2015 and 20 May 2016 and 3 November 2016 at Khon Kaen University, Thailand. A Randomised Complete Block Design (RCBD) with four replications was used for each planting date. Data recorded were leaf life, fallen leaf ratio, leaf area index (LAI), leaf area duration (LAD), storage root dry weight, total crop dry weight, harvest index (HI) and daily weather data. The results indicated that greater values of leaf life during 180 days after planting (DAP), LAD during 180-270 DAP, and LAI at 270 DAP appeared to associate with higher temperature. A combination of LAI at $180 \mathrm{DAP}$, leaf life during 90 and $180 \mathrm{DAP}$ and fallen leaf ratio for 90 and 180 DAP was related to total biomass, while storage root dry weight was associated with a combination of LAI at 180 and 270 DAP and fallen leaf ratio for 90 and 180 DAP. CMR38-125-77 produced high total biomass and storage root dry weight at 270 DAP with possessing high LAI at 180 DAP.
\end{abstract}

Key words: Dry weight, fallen leaf ratio, leaf area duration, leaf area index, leaf life, temperature

\section{INTRODUCTION}

Cassava (Manihot esculenta Crantz) is a tropical root crop which adapts to a wide range of environments (An et al., 2012) and it is recognized as food, feed and energy crop of the world (Haggblade et al., 2012). As increasing of the population, global cassava demand has also been rising. Storage root yield of cassava depends on leaves which are the main sources for crop photosynthesis (ElSharkawy, 2003) and the persistence of leaves also affects final crop yields (Thomas and Smart, 1993). Planting cassava genotype with appropriate growth of leaf is an alternative management for sustainable agriculture to improve cassava productivity and this involves cassava breeding for proper leaf growth habit to enhance maximum yield. The different yields of cassava genotypes were associated with leaf and type of branching (Tan and Cock, 1979; Ogola and Mathews, 2011), and leaf defoliation during the crop growing period with reducing storage root yield of cassava has been recorded (Page et al., 1980).
Thailand is producing cassava to supply the world market and a crop is normally planted in the early and late rainy seasons with the growing durations vary from 8 to 12 months, cover almost all seasons of tropical savanna agro-climate (winter or cool dry season from mid-October to mid-February, summer or hot dry season from midFebruary to mid-May, and rainy season from mid-May to mid-October). However, average productivity of the whole country is presently lower than the expected yield (Anonymous, 2008). The climatic factors with difficult controlling such as temperature and solar radiation during the growing period affect development and physiological process, as well as yield of cassava. The temperature below $17{ }^{\circ} \mathrm{C}$ or above $37^{\circ} \mathrm{C}$ delayed a sprouting of shoots and leaves of cassava (Keating and Evenson, 1979). The optimum temperature for cassava photosynthesis and growth varies from $25{ }^{\circ} \mathrm{C}$ to $35^{\circ} \mathrm{C}$ (El-Sharkawy et al., 1992). The environmental site with higher total amount of solar radiation during cassava growing period allows more crop growth and yield (Phuntupan and Banterng, 2017). 
Planting cassava with recommended genotypes that perform well to particular growing season could help increase yield productivity level.

A study on leaf performances of cassava genotypes in different growing seasons and its relationship with biomass production provides valuable information for developing the recommendation on suitable cassava genotypes and designing a favorable plant type for breeding program, ultimately could contribute to improve cassava productivity. There are previous research articles that demonstrated leaf performances, growth and yield of cassava (Cock et al., 1979; Irikura et al., 1979; Lahai et al., 1999), but those studies were based on other cassava genotypes, experimental sites and growing environments. The information about leaf growth habit and its relation to storage root yield for cassava genotypes in different growing seasons in Thailand is still limited. The objective of this study was to investigate leaf performances and biomass of the four cassava genotypes grown under irrigated condition in different growing seasons.

\section{MATERIALS AND METHODS}

The experiments were carried on during 2015 to 2017 at the Field Crop Research Station of Khon Kaen University, located in Khon Kaen Province, Thailand $\left(16^{\circ} 28 \mathrm{~N}, 102^{\circ} 48 \mathrm{E}, 200 \mathrm{~m}\right.$ above mean sea level). The soil type was Yasothon series with sandy loam texture (Oxic Paleustult). The four different cassava genotypes were arranged as treatment. Kasetsart 50 genotype (branching type) was released by Kasetsart University, Thailand. Rayong 9 (non-branching type), Rayong 11 (branching type) and CMR38-125-77 (branching type) were introduced by Department of Agriculture, Thailand.

Before planting, conventional tillage for cassava experiment was done. The stems for the four different genotypes were harvested from the same growing area at 270 days after planting (DAP) and cut as stakes at $20 \mathrm{~cm}$ in length, and then soaked with thiamethoxam $25 \%$ water dispersible granules at a rate of 4 grams per 20 liters of water for 30 minutes to protect against cassava mealy bug (Anonymous, 2008). The four cassava genotypes were planted at 30 June 2015 and 20 May 2016 (early rainy season with warm temperature at the beginning) and at 5 October 2015 and 3 November 2016 (late rainy season with cool temperature at the beginning). Each planting date had a Randomized Complete Block Design (RCBD) with four replications. Plot size was $7 \times 4 \mathrm{~m}$ with a spacing of $100 \times 100 \mathrm{~cm}$. Weeds were controlled by hand throughout the experimental fields. At $30 \mathrm{DAP}, \mathrm{KCl}$ fertiliser was applied based on soil properties that were determined before planting and cassava nutrient requirements proposed by Howeler (2002), and sufficient concentration of available phosphorus for cassava caused without phosphorus application. In addition, $(\mathrm{NH})_{2} \mathrm{SO}_{4}$ fertiliser was applied at a rate of $46.9 \mathrm{~kg} \mathrm{ha}^{-1}$ and fertilisation of $\mathrm{N}-\mathrm{P}_{2} \mathrm{O}_{5}-\mathrm{K}_{2} \mathrm{O}$ formula 15-0-18 was applied at the rate of $312.5 \mathrm{~kg} \mathrm{ha}^{-1}$ at $60 \mathrm{DAP}$ (Anonymous, 2008). The sets of tensiometer were placed at depth of 40 $\mathrm{cm}$ for all planting dates to monitor soil water tension.
Supplementary irrigation was applied to avoid crop water stress with an overhead sprinkler system when water tension was below $-30 \mathrm{kPa}$ and irrigation was terminated when the water tension was between -20 and $-10 \mathrm{kPa}$.

\section{Data collection}

Leaf life spans for ten consecutive leaves (starting from 90 DAP) for the four non-destructive plants in each experimental plot were measured by counting the time from tagging the beginning fully expanded leaf to leaf fall (Alves, 2002) and then averaged, and means for ten consecutive leaf life were also recorded again from the same four non-destructive plants with starting from 180 DAP. Fallen leaves were collected from sampled plants every 7 days (starting from emergence date to 270 DAP), and those sampled plants were harvested at 90,180 and 270 DAP. The harvested plants were separated into leaves (green leaves), storage root and other organs. Fallen leaves and all plant materials were then subsampled (about $10 \%$ of the total fresh weight of each organ). Subsamples of green leaves were used to measure leaf area using a leaf area meter (LI-3100, LI-Cor, Inc., USA). To determine dry weight, all subsamples were oven-dried at $80{ }^{\circ} \mathrm{C}$ until constant weight. The ratio of canopy leaf area to ground area or leaf area index (LAI) at 90, 180 and 270 DAP were recorded. The values of leaf area duration (LAD) between 90-180 and 180-270 DAP were calculated by following $\left[\left(\mathrm{L}_{2}+\mathrm{L}_{1}\right) / 2\right] \times\left[\left(\mathrm{T}_{2}-\mathrm{T}_{1}\right)\right]$, where $\mathrm{L}_{1}$ and $L_{2}$ stand for leaf area at time $T_{1}$ and $T_{2}$, respectively. The fallen leaf ratios were determined as a proportion of accumulated fallen leaf dry weight to accumulated total leaf dry weight for 90, 180 and 270 DAP. The harvest index (HI) values at 90, 180 and 270 DAP were recorded as a ratio of storage root dry weight to total crop dry weight. In addition, daily rainfall, maximum and minimum temperatures and solar radiation were also observed during the experimental periods.

\section{Statistical analysis}

Analysis of variance (ANOVA) for each experiment or planting date, combined analysis for all planting dates and mean comparisons based on least significant difference test (LSD) were performed by following the procedure of Gomez and Gomez (1984) and by using MSTAT-C Version 1.42 package (Freed and Nissen, 1992). Stepwise regression analysis was done to examine the relationship between leaf characteristics and biomass by using Statistix 10 program (Analytical software, 2013).

\section{RESULTS AND DISCUSSION}

The information on leaf performances for different cassava genotypes grown under different seasons in the tropical savanna agro-climate are very useful for preparing the recommendation on suitable crop management practices, designing plant types and improving cassava genotype for maximum yield. 


\section{Variation of planting date, genotype and planting date $\times$ genotype}

The results from combined analysis of variance showed highly significant effects $(p<0.01)$ of planting date, genotype and planting date $\times$ genotype for all crop traits (Table 1). The variation due to planting date shared the large proportion of total variation for almost all crop traits as compared to the variation for genotype and planting date $\times$ genotype. This demonstrated that climatic factors such as temperature and solar radiation in different planting dates had more impact to most recorded crop traits (Figure 1), while rainfall might not be a limiting factor for cassava growth in this study, because plants were taken care with supplementary irrigation during the dry periods. Genotype contributed the highest variation for leaf life during $90 \mathrm{DAP}$ and $\mathrm{HI}$ at $270 \mathrm{DAP}$, indicating more effect of genotype on these traits than planting date and planting date $\times$ genotype, and this corresponds to a report of Kawano (1990) who suggested significant classification of cassava genotypes based on HI. A significant interaction between planting date and genotype indicates different responses of the three cassava genotypes in the four planting dates and the highest variations due to planting date $\times$ genotype were also recorded for LAD during 180-270 DAP, LAI at 180 DAP, fallen leaf ratio for 90 and 270 DAP and total dry weight at 90 DAP.

\section{Leaf performances}

This is the first report on leaf life during 90 and 180 DAP for the four cassava genotypes grown under different seasons in Thailand. Although Irikura et al. (1979) used to report leaf life of cassava, but that was only for a single growing season at different environments. Cassava leaf life has also been reported by Lahai et al. (1999) but they observed only from 1 to 4 months after planting for a single planting date in each environment. The results in Table 2 revealed that Kasetsart 50 had the longest leaf life during 90 and 180 DAP when compared to the other three genotypes $(\mathrm{p}<0.01)$. Kasetsart 50 planted on the 30 June 2015 and the 5 October 2015 had the longest leaf life during 90 DAP ( $p<0.01)$, while Kasetsart 50 planted on the 5 October 2015 had the longest leaf life during 180 DAP $(p<0.01)$. The planting date of 5 October 2015 gave the longest leaf life for both during 90 and 180 DAP (p < 0.01 ). During 180 DAP of the 5 October 2015 and the 3 November 2016 planting dates, longer leaf life might be due to higher temperature and solar radiation during leaf development (Table 2) and in the time of 180-270 DAP (Figure 1). Leaf life of cassava normally depends on genotype, shade level and abiotic stress such as water and temperature (Cock et al., 1979; Irikura et al., 1979). Since the optimum average temperature for cassava growth has been reported and varied from 25 to $29^{\circ} \mathrm{C}$ (Alves, 2002), maximum temperature during our growing period which is higher than optimal threshold might causes longer cassava leaf life. However, a study of Irikura et al. (1979) about leaf life of cassava grown under three different temperatures $\left(20,24\right.$ and $\left.28{ }^{\circ} \mathrm{C}\right)$ showed that higher temperature decreased cassava leaf life. The different results between our study and a previous study might be due to differences in tested genotypes, cultural practices and environmental conditions.

The values for LAD during the three growing periods of the four cassava genotypes planted in the four planting dates were shown in Table 3. Kasetsart 50 genotype had the highest LAD during 90-180 DAP (p < 0.01) and 180270 DAP $(p<0.01)$. Kasetsart 50 planted on the 30 June 2015 produced the maximum LAD in 90-180 DAP ( $\mathrm{p}<$ 0.01). During 180-270 DAP, Kasetsart 50 planted on the 30 June 2015 and the 3 November 2016 had the highest results in LAD ( $p<0.01)$. The planting date of 30 June 2015 had the highest LAD during the phase of 90-180 DAP ( $\mathrm{p}<0.01$ ), while the 5 October 2015 and the 3 November 2016 planting dates had the highest results in LAD for the 180-270 DAP ( $p<0.01$ ). Higher average temperature during 180-270 DAP for the planting dates of 5 October 2015 and 3 November 2016 (Figure 1), therefore, did not only increase leaf life, but also might be a reason for higher LAD values.

Based on LAI values (Table 3), CMR38-125-77 had the highest LAI at 90 DAP ( $\mathrm{p}<0.01$ ). Rayong 9 and CMR38-125-77 gave the highest results at 180 DAP ( $\mathrm{p}<$ 0.01). At 270 DAP, Kasetsart 50 and CMR38-125-77 had the highest LAI values $(\mathrm{p}<0.01)$. The planting date of 30 June 2015 gave the highest result in LAI at 90 DAP ( $p<$ 0.01). The 30 June 2015 and the 20 May 2016 planting dates had the highest LAI values for 180 DAP and the 5 October 2015 and the 3 November 2016 for the 270 DAP ( $p<0.01$ ). CMR38-125-77 planted on the 30 June 2015 had the highest result in LAI at 90 DAP $(p<0.01)$. At 180 DAP, Kasetsart 50 planted on the 5 October 2015, Rayong 9 at 30 June 2015 and Rayong 11 at the 5 October 2015 had the highest LAI values $(\mathrm{p}<0.01)$. CMR38-125-77 planted on the 3 November 2016 gave the highest LAI value at 270 DAP $(\mathrm{p}<0.01)$. This study also revealed moderate association between the LAI values at 180 and 270 and LAD values during 90-180 and 180-270 DAP with the correlation coefficient of 0.5 ( $p<0.01$ ). Based on a study with eddoe (Colocasia esculenta) which is also a kind of tuber crop, LAI values were positively associated with the values of LAD (Roychowdhury, 1995) The correlation coefficient between LAI at 90 and 180 DAP and leaf life during 90 and 180 DAP was significant with the value of $0.5(\mathrm{p}<0.01)$. Higher correlation between these leaf traits, however, might be found for the study with various and large number of tested cassava genotypes. 
Table 1. Mean squares obtained from combined analysis for leaf life, leaf area duration (LAD), leaf area index (LAI), fallen leaf ratio, total dry weight, storage root dry weight and harvest index (HI) of the four cassava genotypes grown at the four planting dates

\begin{tabular}{|c|c|c|c|c|c|c|c|c|c|c|}
\hline \multirow{2}{*}{ Crop trait } & \multicolumn{10}{|c|}{ Source variation } \\
\hline & \multicolumn{2}{|c|}{ Planting $(\mathbf{P})$} & \multicolumn{2}{|c|}{ Replication/P } & \multicolumn{2}{|c|}{ Genotype (G) } & \multicolumn{2}{|c|}{$\mathbf{G} \times \mathbf{P}$} & \multicolumn{2}{|c|}{ Pool error } \\
\hline Leaf life during 90 DAP (days) & 970 & $(4.61)^{* *}$ & 60 & $(0.28)$ & 15774 & $(75.05)^{* *}$ & 3986 & $(18.97)^{* *}$ & 227 & $(1.08)$ \\
\hline Leaf life during 180 DAP (days) & 74648 & $(67.28)^{* *}$ & 52 & $(0.05)$ & 14002 & $(12.62)^{* *}$ & 22066 & $(19.89)^{* *}$ & 184 & $(0.17)$ \\
\hline LAD during 90-180 DAP ( $\mathrm{m}^{2}$ day) & 45156 & $(42.44)^{* *}$ & 1617 & $(6.08)$ & 23431 & $(22.02)^{* *}$ & 8796 & $(24.80)^{* *}$ & 413 & $(4.66)$ \\
\hline LAD during $180-270$ DAP ( $\mathrm{m}^{2}$ day) & 24380 & $(16.55)^{* *}$ & 1282 & $(3.48)$ & 39356 & $(26.72)^{* *}$ & 23854 & $(48.58) * *$ & 573 & $(4.67)$ \\
\hline LAI at $90\left(\mathrm{~cm}^{2} \mathrm{~cm}^{-2}\right)$ & 28.11 & $(70.25)^{* *}$ & 0.01 & $(0.12)$ & 2.50 & $(6.23)^{* *}$ & 3.10 & $(23.19)^{* *}$ & 0.01 & $(0.21)$ \\
\hline LAI at $180\left(\mathrm{~cm}^{2} \mathrm{~cm}^{-2}\right)$ & 15.80 & $(19.01)^{* *}$ & 0.64 & $(0.77)$ & 1.22 & $(1.47)^{* *}$ & 64.99 & $(78.17)^{* *}$ & 0.48 & $(0.58)$ \\
\hline LAI at $270\left(\mathrm{~cm}^{2} \mathrm{~cm}^{-2}\right)$ & 108.65 & $(52.78)^{* *}$ & 0.33 & $(0.16)$ & 36.54 & $(17.75)^{* *}$ & 58.46 & $(28.40)^{* *}$ & 1.89 & $(0.92)$ \\
\hline Fallen leaf ratio for 90 DAP & 0.039 & $(42.38)^{* *}$ & 0.003 & $(3.29)$ & 0.003 & $(3.40)^{* *}$ & 0.039 & $(42.58)^{* *}$ & 0.008 & $(8.35)$ \\
\hline Fallen leaf ratio for $180 \mathrm{DAP}$ & 0.99 & $(62.86)^{* *}$ & 0.00 & $(0.07)$ & 0.09 & $(5.83)^{* *}$ & 0.41 & $(25.86)^{* *}$ & 0.09 & $(5.37)$ \\
\hline Fallen leaf ratio for $270 \mathrm{DAP}$ & 0.23 & $(36.49)^{* *}$ & 0.01 & $(1.70)$ & 0.11 & $(17.83)^{* *}$ & 0.25 & $(39.61)^{* *}$ & 0.03 & $(4.37)$ \\
\hline Total dry weight at 90 DAP $\left(\right.$ g plant $\left.^{-1}\right)$ & 57849 & $(15.31)^{* *}$ & 3956 & $(1.05)$ & 148107 & $(39.20)^{* *}$ & 156239 & $(41.35)^{* *}$ & 11685 & $(3.09)$ \\
\hline Total dry weight at 180 DAP $\left(\right.$ g plant $\left.^{-1}\right)$ & 13780000 & $(75.14)^{* *}$ & 76309 & $(0.42)$ & 1917441 & $(10.46)^{* *}$ & 2379263 & $(12.97)^{* *}$ & 184997 & $(1.01)$ \\
\hline Total dry weight at 270 DAP $\left(\mathrm{g} \mathrm{plant}^{-1}\right)$ & 13340000 & $(63.86)^{* *}$ & 83615 & $(0.40)$ & 1741736 & $(8.34)^{* *}$ & 5333792 & $(25.53)^{* *}$ & 384767 & $(1.84)$ \\
\hline Storage root dry weight at 90 DAP $\left(\mathrm{g} \mathrm{plant}^{-1}\right)$ & 92915 & $(45.36)^{* *}$ & 761 & $(0.37)$ & 44450 & $(21.70)^{* *}$ & 64136 & $(31.31)^{* *}$ & 2567 & $(1.25)$ \\
\hline Storage root dry weight at $180 \mathrm{DAP}\left(\mathrm{g} \mathrm{plant}^{-1}\right)$ & 7069247 & $(85.53)^{* *}$ & 89068 & $(1.08)$ & 164384 & $(1.99)^{* *}$ & 785290 & $(9.50)^{* *}$ & 157222 & $(1.90)$ \\
\hline Storage root dry weight at 270 DAP $\left(\mathrm{g}\right.$ plant $\left.^{-1}\right)$ & 2936893 & $(42.22)^{* *}$ & 41483 & $(0.60)$ & 988267 & $(14.21)^{* *}$ & 2811368 & $(40.42)^{* *}$ & 177611 & $(2.55)$ \\
\hline $\mathrm{HI}$ at $90 \mathrm{DAP}$ & 2.731 & $(86.02)^{* *}$ & 0.005 & $(0.17)$ & 0.050 & $(1.57)^{* *}$ & 0.372 & $(11.71)^{* *}$ & 0.017 & $(0.54)$ \\
\hline HI at 180 DAP & 0.583 & $(66.86)^{* *}$ & 0.003 & $(0.37)$ & 0.066 & $(7.55)^{* *}$ & 0.205 & $(23.46)^{* *}$ & 0.015 & $(1.76)$ \\
\hline HI at 270 DAP & 0.178 & $(32.60)^{* *}$ & 0.003 & $(0.56)$ & 0.220 & $(40.32)^{* *}$ & 0.135 & $(24.75)^{* *}$ & 0.010 & (1.77) \\
\hline
\end{tabular}




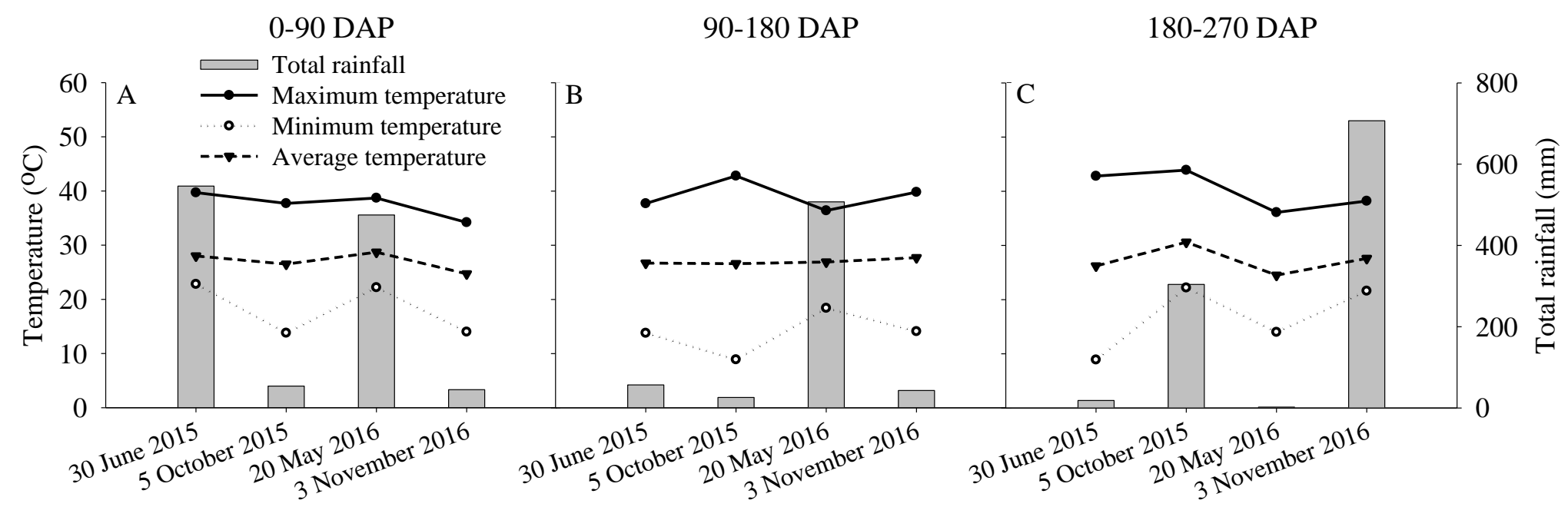

Planting date

Planting date

Planting date

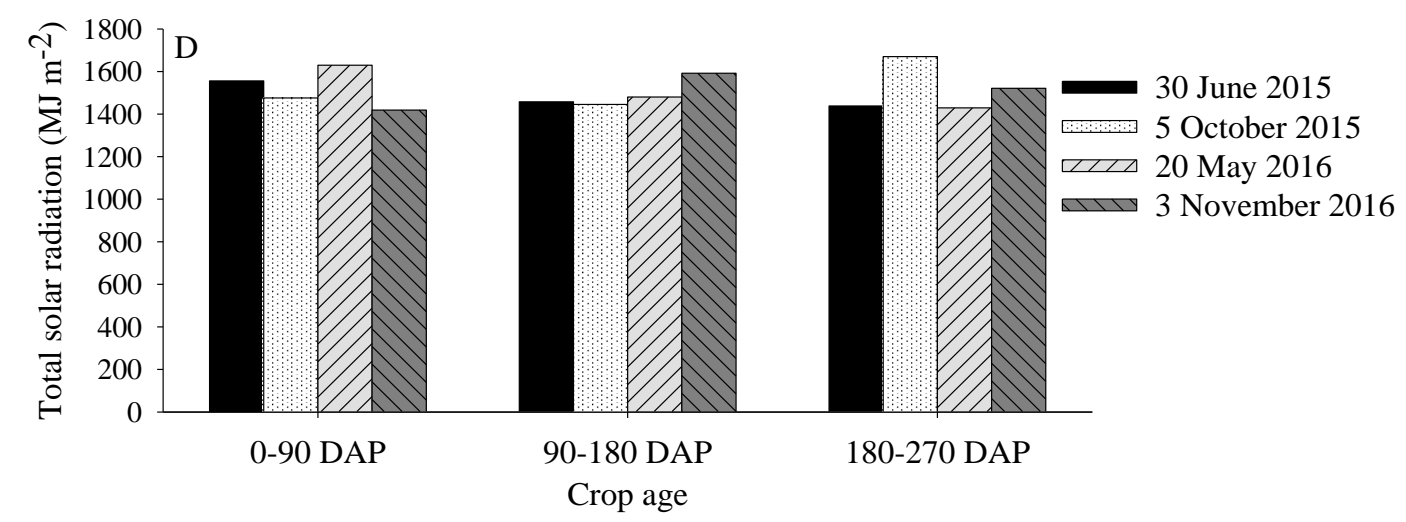

Figure 1. Maximum temperature $\left({ }^{\circ} \mathrm{C}\right)$, minimum temperature $\left({ }^{\circ} \mathrm{C}\right)$, average temperature $\left({ }^{\circ} \mathrm{C}\right)$ and total rainfall $(\mathrm{mm})$ during $0-90(\mathrm{~A}), 90-180(\mathrm{~B})$ and $180-270(\mathrm{C})$ days after planting $(\mathrm{DAP})$ and total solar radiation $\left(\mathrm{D}, \mathrm{MJ} \mathrm{m}^{-2}\right)$ for three different crop durations for the four planting dates. 
Table 2. Leaf life during 90 and 180 days after planting (DAP) of the four cassava genotypes grown at the four planting dates and maximum temperature $\left({ }^{\circ} \mathrm{C}\right)$, minimum temperature $\left({ }^{\circ} \mathrm{C}\right)$, average temperature $\left({ }^{\circ} \mathrm{C}\right)$ and average solar radiation $\left(\mathrm{MJ} \mathrm{m}^{-2}\right.$ day $\left.^{-1}\right)$ for leaf life during 90 and 180 DAP

\begin{tabular}{|c|c|c|c|c|c|c|c|c|c|c|}
\hline \multirow{2}{*}{ Factor } & \multirow{2}{*}{$\begin{array}{c}\text { Leaf life } \\
\text { (days) } \\
\text { during } \\
90 \text { DAP }\end{array}$} & \multicolumn{3}{|c|}{ Temperature $\left({ }^{\circ} \mathrm{C}\right)$} & \multirow{2}{*}{$\begin{array}{c}\text { Average solar } \\
\text { radiation } \\
\left(\mathrm{MJ} \mathrm{m}^{-2} \text { day }^{-1}\right)\end{array}$} & \multirow{2}{*}{$\begin{array}{c}\text { Leaf life } \\
\text { (days) } \\
\text { during } \\
\text { 180 DAP }\end{array}$} & \multicolumn{3}{|c|}{ Temperature $\left({ }^{\circ} \mathrm{C}\right)$} & \multirow{2}{*}{$\begin{array}{c}\text { Average solar } \\
\text { radiation } \\
\left(\mathbf{M J ~ m}^{-2} \text { day }^{-1}\right)\end{array}$} \\
\hline & & Maximum & Minimum & Average & & & Maximum & Minimum & Average & \\
\hline Kasetsart 50 (G1) & $88^{\mathrm{A}}$ & & & & & $104^{\mathrm{A}}$ & & & & \\
\hline Rayong 9 (G2) & $55^{\mathrm{C}}$ & & & & & $71^{\mathrm{C}}$ & & & & \\
\hline Rayong $11(\mathrm{G} 3)$ & $45^{\mathrm{D}}$ & & & & & $66^{\mathrm{D}}$ & & & & \\
\hline CMR38-125-77 (G4) & $65^{\mathrm{B}}$ & & & & & $77^{\mathrm{B}}$ & & & & \\
\hline 30 June $2015(\mathrm{P} 1)$ & $59^{\mathrm{C}}$ & 37.3 & 19.5 & 27.1 & 16.3 & $46^{\mathrm{C}}$ & 38.7 & 8.9 & 24.2 & 15.2 \\
\hline 5 October $2015(\mathrm{P} 2)$ & $69^{\mathrm{A}}$ & 42.3 & 8.9 & 25.3 & 16.0 & $120^{\mathrm{A}}$ & 43.9 & 22.2 & 30.0 & 18.3 \\
\hline 20 May 2016 (P3) & $65^{\mathrm{B}}$ & 36.5 & 22.5 & 27.4 & 16.1 & $45^{\mathrm{C}}$ & 34.2 & 14.0 & 24.3 & 15.0 \\
\hline 3 November $2016(\mathrm{P} 4)$ & $60^{\mathrm{C}}$ & 39.5 & 14.1 & 26.4 & 17.0 & $107^{\mathrm{B}}$ & 39.3 & 21.6 & 27.6 & 16.4 \\
\hline $\mathrm{G} 1 \times \mathrm{P} 1$ & $95^{\mathrm{A}}$ & & & & & $43^{\mathrm{IJK}}$ & & & & \\
\hline $\mathrm{G} 1 \times \mathrm{P} 2$ & $95^{\mathrm{A}}$ & & & & & $180^{\mathrm{A}}$ & & & & \\
\hline $\mathrm{G} 1 \times \mathrm{P} 3$ & $88^{\mathrm{B}}$ & & & & & $40^{\mathrm{K}}$ & & & & \\
\hline $\mathrm{G} 1 \times \mathrm{P} 4$ & $73^{\mathrm{CD}}$ & & & & & $154^{\mathrm{B}}$ & & & & \\
\hline $\mathrm{G} 2 \times \mathrm{P} 1$ & $47^{\mathrm{I}}$ & & & & & $55^{\mathrm{G}}$ & & & & \\
\hline $\mathrm{G} 2 \times \mathrm{P} 2$ & $58^{\mathrm{FG}}$ & & & & & $84^{\mathrm{F}}$ & & & & \\
\hline $\mathrm{G} 2 \times \mathrm{P} 3$ & $52^{\mathrm{HI}}$ & & & & & $51^{\mathrm{GH}}$ & & & & \\
\hline $\mathrm{G} 2 \times \mathrm{P} 4$ & $64^{\mathrm{E}}$ & & & & & $92^{\mathrm{DE}}$ & & & & \\
\hline $\mathrm{G} 3 \times \mathrm{P} 1$ & $33^{\mathrm{J}}$ & & & & & $42^{\mathrm{JK}}$ & & & & \\
\hline $\mathrm{G} 3 \times \mathrm{P} 2$ & $49^{I}$ & & & & & $91^{\mathrm{E}}$ & & & & \\
\hline $\mathrm{G} 3 \times \mathrm{P} 3$ & $63^{\mathrm{E}}$ & & & & & $47^{\mathrm{HI}}$ & & & & \\
\hline $\mathrm{G} 3 \times \mathrm{P} 4$ & $36^{\mathrm{J}}$ & & & & & $85^{\mathrm{F}}$ & & & & \\
\hline $\mathrm{G} 4 \times \mathrm{P} 1$ & $60^{\mathrm{EF}}$ & & & & & $45^{\mathrm{IJ}}$ & & & & \\
\hline $\mathrm{G} 4 \times \mathrm{P} 2$ & $74^{\mathrm{C}}$ & & & & & $126^{\mathrm{C}}$ & & & & \\
\hline $\mathrm{G} 4 \times \mathrm{P} 3$ & $55^{\mathrm{GH}}$ & & & & & $42^{\mathrm{JK}}$ & & & & \\
\hline $\mathrm{G} 4 \times \mathrm{P} 4$ & $69^{\mathrm{D}}$ & & & & & $95^{\mathrm{D}}$ & & & & \\
\hline $\mathrm{CV}(\%)$ & 3.98 & & & & & 2.85 & & & & \\
\hline
\end{tabular}


Table 3. Leaf area duration (LAD) during 90-180 and 180-270 days after planting (DAP), leaf area index (LAI) at 90, 180 and 270 DAP and fallen leaf ratio for 90 , 180 and 270 DAP of the four cassava genotypes grown at the four planting dates

\begin{tabular}{|c|c|c|c|c|c|c|c|c|}
\hline \multirow[b]{2}{*}{ Factor } & \multicolumn{2}{|c|}{ LAD (m² day) } & \multicolumn{3}{|c|}{ LAI $\left(\mathrm{cm}^{2} \mathbf{c m}^{-2}\right)$} & \multicolumn{3}{|c|}{ Fallen leaf ratio } \\
\hline & $\begin{array}{c}\text { 90-180 } \\
\text { DAP }\end{array}$ & $\begin{array}{c}\text { 180-270 } \\
\text { DAP }\end{array}$ & $\begin{array}{c}90 \\
\text { DAP }\end{array}$ & $\begin{array}{c}180 \\
\text { DAP }\end{array}$ & $\begin{array}{c}270 \\
\text { DAP }\end{array}$ & $\begin{array}{c}90 \\
\text { DAP }\end{array}$ & $\begin{array}{c}180 \\
\text { DAP }\end{array}$ & $\begin{array}{l}270 \\
\text { DAP }\end{array}$ \\
\hline Kasetsart $50(\mathrm{G} 1)$ & $264.4^{\mathrm{A}}$ & $311.0^{\mathrm{A}}$ & $1.3^{\mathrm{B}}$ & $3.5^{\mathrm{B}}$ & $4.1^{\mathrm{A}}$ & $0.08^{\mathrm{A}}$ & $0.31^{\mathrm{B}}$ & $0.62^{\mathrm{BC}}$ \\
\hline Rayong 9 (G2) & $185.7^{\mathrm{C}}$ & $191.4^{\mathrm{C}}$ & $0.9^{\mathrm{C}}$ & $3.8^{\mathrm{A}}$ & $2.2^{\mathrm{C}}$ & $0.06^{\mathrm{B}}$ & $0.08^{\mathrm{D}}$ & $0.60^{\mathrm{C}}$ \\
\hline Rayong 11 (G3) & $180.9^{\mathrm{C}}$ & $257.2^{\mathrm{B}}$ & $1.2^{\mathrm{B}}$ & $3.6^{\mathrm{B}}$ & $3.3^{\mathrm{B}}$ & $0.07^{\mathrm{AB}}$ & $0.25^{\mathrm{C}}$ & $0.64^{\mathrm{B}}$ \\
\hline CMR38-125-77 (G4) & $212.2^{\mathrm{B}}$ & $236.8^{\mathrm{B}}$ & $1.9^{\mathrm{A}}$ & $3.8^{\mathrm{A}}$ & $3.9^{\mathrm{A}}$ & $0.08^{\mathrm{A}}$ & $0.43^{\mathrm{A}}$ & $0.71^{\mathrm{A}}$ \\
\hline 30 June 2015 (P1) & $280.8^{\mathrm{A}}$ & $246.2^{\mathrm{B}}$ & $2.4^{\mathrm{A}}$ & $4.0^{\mathrm{B}}$ & $2.5^{\mathrm{C}}$ & $0.07^{\mathrm{B}}$ & $0.25^{\mathrm{BC}}$ & $0.69^{\mathrm{A}}$ \\
\hline 5 October $2015(\mathrm{P} 2)$ & $160.6^{\mathrm{C}}$ & $265.8^{\mathrm{AB}}$ & $0.6^{\mathrm{D}}$ & $3.3^{\mathrm{C}}$ & $4.3^{\mathrm{B}}$ & $0.10^{\mathrm{A}}$ & $0.32^{\mathrm{A}}$ & $0.55^{\mathrm{C}}$ \\
\hline 20 May $2016(\mathrm{P} 3)$ & $180.1^{\mathrm{BC}}$ & $196.4^{\mathrm{C}}$ & $0.7^{\mathrm{C}}$ & $4.3^{\mathrm{A}}$ & $1.8^{\mathrm{D}}$ & $0.08^{\mathrm{B}}$ & $0.27^{\mathrm{AB}}$ & $0.70^{\mathrm{A}}$ \\
\hline 3 November 2016 (P4) & $221.8^{\mathrm{B}}$ & $287.9^{\mathrm{A}}$ & $1.6^{\mathrm{B}}$ & $3.1^{\mathrm{C}}$ & $5.0^{\mathrm{A}}$ & $0.04^{\mathrm{C}}$ & $0.22^{\mathrm{C}}$ & $0.65^{\mathrm{B}}$ \\
\hline $\mathrm{G} 1 \times \mathrm{P} 1$ & $407.2^{\mathrm{A}}$ & $415.8^{\mathrm{A}}$ & $2.0^{\mathrm{C}}$ & $2.1^{\mathrm{H}}$ & $3.9^{\mathrm{E}}$ & $0.10^{\mathrm{BC}}$ & $0.53^{\mathrm{A}}$ & $0.61^{\mathrm{F}}$ \\
\hline $\mathrm{G} 1 \times \mathrm{P} 2$ & $220.3^{\mathrm{CDE}}$ & $277.0^{\mathrm{CDE}}$ & $0.6^{\mathrm{H}}$ & $4.8^{\mathrm{AB}}$ & $4.6^{\mathrm{D}}$ & $0.13^{\mathrm{A}}$ & $0.41^{\mathrm{BC}}$ & $0.52^{\mathrm{G}}$ \\
\hline $\mathrm{G} 1 \times \mathrm{P} 3$ & $258.0^{\mathrm{BC}}$ & $170.9^{\mathrm{HI}}$ & $0.9^{\mathrm{G}}$ & $4.7^{\mathrm{B}}$ & $1.9^{\mathrm{GH}}$ & $0.06^{\mathrm{DE}}$ & $0.26^{\mathrm{DE}}$ & $0.67^{\mathrm{CDE}}$ \\
\hline $\mathrm{G} 1 \times \mathrm{P} 4$ & $237.3^{\mathrm{CD}}$ & $380.2^{\mathrm{A}}$ & $1.6^{\mathrm{D}}$ & $4.4^{\mathrm{C}}$ & $6.2^{\mathrm{B}}$ & $0.02^{\mathrm{F}}$ & $0.10^{\mathrm{GH}}$ & $0.68^{\mathrm{CD}}$ \\
\hline $\mathrm{G} 2 \times \mathrm{P} 1$ & $195.1^{\mathrm{EFG}}$ & $172.7^{\mathrm{GHI}}$ & $1.3^{\mathrm{E}}$ & $5.0^{\mathrm{A}}$ & $1.6^{\mathrm{H}}$ & $0.07^{\mathrm{CD}}$ & $0.47^{\mathrm{AB}}$ & $0.68^{\mathrm{CD}}$ \\
\hline $\mathrm{G} 2 \times \mathrm{P} 2$ & $159.8^{\mathrm{G}}$ & $227.7^{\mathrm{FG}}$ & $0.5^{\mathrm{H}}$ & $3.4^{\mathrm{E}}$ & $3.0^{\mathrm{F}}$ & $0.04^{\mathrm{EF}}$ & $0.32^{\mathrm{CD}}$ & $0.51^{\mathrm{G}}$ \\
\hline $\mathrm{G} 2 \times \mathrm{P} 3$ & $92.3^{\mathrm{H}}$ & $213.0^{\mathrm{FGH}}$ & $0.7^{\mathrm{H}}$ & $1.1^{\mathrm{I}}$ & $2.2^{\mathrm{G}}$ & $0.09^{\mathrm{BC}}$ & $0.20^{\mathrm{EF}}$ & $0.60^{\mathrm{F}}$ \\
\hline $\mathrm{G} 2 \times \mathrm{P} 4$ & $195.1^{\mathrm{EFG}}$ & $152.0^{\mathrm{I}}$ & $1.1^{\mathrm{F}}$ & $3.5^{\mathrm{DE}}$ & $1.8^{\mathrm{GH}}$ & $0.03^{\mathrm{EF}}$ & $0.10^{\mathrm{GH}}$ & $0.63^{\mathrm{DEF}}$ \\
\hline $\mathrm{G} 3 \times \mathrm{P} 1$ & $177.9^{\mathrm{FG}}$ & $257.7^{\mathrm{DEF}}$ & $2.4^{\mathrm{B}}$ & $3.2^{\mathrm{F}}$ & $2.9^{\mathrm{F}}$ & $0.05^{\mathrm{DEF}}$ & $0.44^{\mathrm{B}}$ & $0.70^{\mathrm{C}}$ \\
\hline $\mathrm{G} 3 \times \mathrm{P} 2$ & $199.2^{\mathrm{DEF}}$ & $235.6^{\mathrm{EF}}$ & $0.5^{\mathrm{H}}$ & $4.8^{\mathrm{AB}}$ & $4.4^{\mathrm{D}}$ & $0.13^{\mathrm{A}}$ & $0.31^{\mathrm{D}}$ & $0.63^{\mathrm{DEF}}$ \\
\hline $\mathrm{G} 3 \times \mathrm{P} 3$ & $175.3^{\mathrm{FG}}$ & $235.8^{\mathrm{EF}}$ & $0.6^{\mathrm{H}}$ & $4.7^{\mathrm{B}}$ & $1.5^{\mathrm{H}}$ & $0.04^{\mathrm{EF}}$ & $0.16^{\mathrm{FG}}$ & $0.60^{\mathrm{F}}$ \\
\hline $\mathrm{G} 3 \times \mathrm{P} 4$ & $168.1^{\mathrm{FG}}$ & $299.7^{\mathrm{BCD}}$ & $1.4^{\mathrm{E}}$ & $4.4^{\mathrm{C}}$ & $4.5^{\mathrm{D}}$ & $0.05^{\mathrm{DE}}$ & $0.06^{\mathrm{H}}$ & $0.62^{\mathrm{EF}}$ \\
\hline $\mathrm{G} 4 \times \mathrm{P} 1$ & $277.3^{\text {B }}$ & $138.6^{\mathrm{I}}$ & $3.9^{\mathrm{A}}$ & $3.7^{\mathrm{D}}$ & $1.5^{\mathrm{H}}$ & $0.05^{\mathrm{DEF}}$ & $0.42^{\mathrm{B}}$ & $0.76^{\mathrm{B}}$ \\
\hline $\mathrm{G} 4 \times \mathrm{P} 2$ & $163.5^{\mathrm{FG}}$ & $323.1^{\mathrm{B}}$ & $0.6^{\mathrm{H}}$ & $2.1^{\mathrm{H}}$ & $5.4^{\mathrm{C}}$ & $0.11^{\mathrm{AB}}$ & $0.30^{\mathrm{D}}$ & $0.52^{\mathrm{G}}$ \\
\hline $\mathrm{G} 4 \times \mathrm{P} 3$ & $198.2^{\mathrm{D}-\mathrm{G}}$ & $165.8^{\mathrm{I}}$ & $0.7^{\mathrm{H}}$ & $3.7^{\mathrm{B}}$ & $1.5^{\mathrm{H}}$ & $0.11^{\mathrm{AB}}$ & $0.12^{\mathrm{FGH}}$ & $0.91^{\mathrm{A}}$ \\
\hline $\mathrm{G} 4 \times \mathrm{P} 4$ & $248.2^{\mathrm{BC}}$ & $319.6^{\mathrm{BC}}$ & $2.3^{\mathrm{B}}$ & $2.9^{\mathrm{G}}$ & $7.3^{\mathrm{A}}$ & $0.04^{\mathrm{EF}}$ & $0.06^{\mathrm{H}}$ & $0.65^{\mathrm{C}-\mathrm{F}}$ \\
\hline CV (\%) & 9.64 & 9.61 & 6.57 & 3.16 & 6.77 & 20.70 & 18.18 & 4.27 \\
\hline
\end{tabular}

$\mathrm{CV}=$ Coefficient of variation, Values followed by the same letter are not significantly different by least significant difference test (at 0.01 probability level). 
The results in Table 3 also showed that the highest values of fallen leaf ratio were recorded for Kasetsart 50 and CMR38-125-77 for 90 DAP ( $<<0.01)$ and CMR38125-77 for 180 DAP ( $<<0.01)$ and 270 DAP ( $<<0.01)$. The 5 October 2015 planting date had the highest value of fallen leaf ratio for 90 DAP $(p<0.01)$. Whereas, the highest values of fallen leaf ratio for 180 and 270 DAP were observed at the planting dates of 5 October 2015 and 20 May 2016 ( $<$ 0.01) and 30 June 2015 and 20 May 2016 ( p < 0.01), respectively. Kasetsart 50 planted on the 5 October 2015, Rayong 11 at the 5 October 2015 and CMR38-125-77 at the 5 October 2015 and the 20 May 2016 showed the highest fallen leaf ratio for 90 DAP ( $\mathrm{p}<$ 0.01). Kasetsart 50 and Rayong 9 at the 30 June 2015 planting date had the highest fallen leaf ratio for 180 DAP ( $p<0.01$ ) and CMR38-125-77 at the 20 May 2016 for 270 DAP gave the highest fallen leaf ratio $(\mathrm{p}<0.01)$. The values of fallen leaf ratio for 90 DAP were generally smaller than 180 and 270 DAP for all cassava genotypes and planting dates, indicates a larger portion of leaf discarding in the middle and the late growing periods of cassava. This is probably due to leaf senescence and shading effect. The older cassava has bigger shrub that causes less chance for light interception and photosynthesis for lower leaves, and ultimately leaves discarding (Cock et al., 1979; Alves, 2002).

\section{Biomass and its relationship to leaf characteristics}

It could be seen in Table 4 that the highest total dry weight means were observed for CMR38-125-77 at 90 DAP (p < 0.01), Kasetsart 50 at 180 DAP $(\mathrm{p}<0.01)$ and Kasetsart 50 and CMR38-125-77at 270 DAP ( $<<0.01$ ). Rayong 9 and Rayong 11 planted on the 3 November 2016 and CMR38-125-77 at the 5 October 2015 produced the highest total dry weight for 90 DAP ( $p<0.01)$. Kasetsart 50 at the 5 October 2015 planting date had the highest total dry weight for 180 DAP $(\mathrm{p}<0.01)$ and CMR38-125-77 at the 30 June 2015 gave the highest total dry weight value for 270 DAP ( $p<0.01$ ). The 5 October 2015 and the 3 November 2016 planting dates gave the highest total dry weight at 90 DAP ( $p<0.01)$. For 180 and 270 DAP, the highest values of total dry weight were observed at the 20 May 2016 planting date $(\mathrm{p}<0.01)$.

The storage root dry weight means in Table 4 indicated that CMR38-125-77 gave the highest value at 90 DAP ( $\mathrm{p}<0.01$ ) and Kasetsart 50, Rayong 9 and CMR38$125-77$ performed good at 180 DAP $(p<0.01)$. For 270 DAP, CMR38-125-77 had the highest storage root dry weight $(p<0.01)$. The planting date of 5 October 2015 showed the highest storage root dry weight at 90 DAP (p $<0.01$ ), while the 20 May 2016 planting date gave the highest means at 180 and 270 DAP ( $<<0.01$ ). Kasetsart 50 planted on the 3 November 2016 produced the highest storage root dry weight at 90 DAP $(\mathrm{p}<0.01)$. For 180 DAP, Rayong 11 and CMR38-125-77 were good when planted on the 30 June 2015 ( $p<0.01$ ). CMR38-125-77 at the 30 June 2015 had the highest storage root dry weight for 270 DAP $(\mathrm{p}<0.01)$. Based on comparing the planting dates in early rainy season (30 June 2015 and 20 May 2016) and late rainy season (5 October 2015 and 3 November 2016), higher storage root dry weight means at 180 and 270 DAP were recorded for early rainy season planting dates in both 2015 and 2016. The highest values of total biomass and storage root dry weight (3027 and $1750 \mathrm{~g} \mathrm{plant}^{-1}$, respectively) for the 20 May 2016 planting date related with the highest total amount of solar radiation (Figure 1) during the period from planting to 180 DAP (3110 $\left.\mathrm{MJ} \mathrm{m}^{-2}\right)$. Solar radiation is a climatic factor that enhances canopy photosynthesis and biomass accumulation, and higher total amount of solar radiation during growing season in Thailand provided more growth and storage root yield (Phuntupan and Banterng, 2017).

For the values of partitioning capability to storage root or $\mathrm{HI}$ in Table 4, the highest values were found for CMR38-125-77 at 90 DAP ( $p<0.01$ ), Rayong 9 and CMR38-125-77 at 180 DAP $(\mathrm{p}<0.01)$ and Rayong 9 at 270 DAP ( $p<0.01$ ). The 20 May 2016 planting date showed the highest HI for 90 DAP $(\mathrm{p}<0.01)$ and the 5 October 2015 for 180 and 270 DAP ( $p<0.01$ ). Rayong 9 planted on the 3 November 2016 gave the highest HI at 90 DAP ( $\mathrm{p}<0.01$ ). Rayong 9 and Kasetsart 50 performed the best HI for 180 DAP when planted at the 3 November 2016 ( $\mathrm{p}<0.01$ ), while Rayong 9 planted on the 5 October 2015 had the highest mean at 270 DAP ( $p<0.01$ ). The late rainy season planting dates had higher $\mathrm{HI}$ than the early rainy season planting dates.

The stepwise regression analysis given in Table 5 indicated that LAI at 180 DAP, leaf life during 90 and 180 DAP and fallen leaf ratio for 90 and 180 DAP explained total dry weight at 270 DAP with moderate $\mathrm{R}^{2}$ value of 0.6 ( $\mathrm{p}<0.05$ ). The LAI at 180 and 270 DAP and fallen leaf ratio for 90 and 180 DAP described storage root dry weight for 270 DAP with $\mathrm{R}^{2}$ value of 0.5 ( $\mathrm{p}<0.05$ ). Long leaf life of Kasetsart 50 genotype during 90 and 180 DAP (Table 2) related to high total dry weight at 270 DAP (Table 4). However, low partitioning photosynthates from leaf to storage root for Kasetsart 50 genotype (as indicated by small values of $\mathrm{HI}$ at 180 and 270 DAP in Table 4) caused low storage root yield at 270 DAP (Kawano, 1990; Lemoine et al., 2013) and the balance between leaf (source) and storage root (sink) is an essential to maximize yield productivity. Leaf is known as an important source for photosynthetic carbon assimilation and growth (De Tafur et al., 1997; El-Sharkawy, 2003), and cassava has simultaneous growth of leaf area and storage root (Lebot, 2009). Leaf longevity of cassava varies depending on genotypes and it plays an important role to support crop photosynthesis and growth (Cock et al., 1979; Cock, 1984). Lahai et al. (1999) found that leaf lives of three cassava genotypes (80/84, 87/29 and Coco) grown in upland area at Njala (latitude of $8^{\circ} 06$ and longitude of $12^{\circ} 06$ ), Sierra Leone varied from 11 to 84 days and it also had high relationship with storage root yield. 
Table 4. Total dry weight, storage root dry weight and harvest index (HI) at 90, 180 and 270 days after planting (DAP) of the four cassava genotypes grown at the four planting dates

\begin{tabular}{|c|c|c|c|c|c|c|c|c|c|}
\hline \multirow[b]{2}{*}{ Factor } & \multicolumn{3}{|c|}{ Total dry weight $\left(\mathrm{g} \mathrm{plant}^{-1}\right)$} & \multicolumn{3}{|c|}{ Storage root dry weight $\left(\mathrm{g} \mathrm{plant}^{-1}\right)$} & \multicolumn{3}{|c|}{ HI } \\
\hline & $\begin{array}{c}90 \\
\text { DAP }\end{array}$ & $\begin{array}{c}180 \\
\text { DAP }\end{array}$ & $\begin{array}{c}270 \\
\text { DAP }\end{array}$ & $\begin{array}{c}90 \\
\text { DAP }\end{array}$ & $\begin{array}{c}180 \\
\text { DAP }\end{array}$ & $\begin{array}{c}270 \\
\text { DAP }\end{array}$ & $\begin{array}{c}90 \\
\text { DAP }\end{array}$ & $\begin{array}{c}180 \\
\text { DAP }\end{array}$ & $\begin{array}{c}270 \\
\text { DAP }\end{array}$ \\
\hline Kasetsart 50 (G1) & $297^{\mathrm{B}}$ & $1741^{\mathrm{A}}$ & $2502^{\mathrm{A}}$ & $111^{\mathrm{B}}$ & $913^{\mathrm{A}}$ & $1264^{\mathrm{D}}$ & $0.46^{\mathrm{B}}$ & $0.65^{\mathrm{B}}$ & $0.56^{\mathrm{C}}$ \\
\hline Rayong 9 (G2) & $244^{\mathrm{C}}$ & $1385^{\mathrm{B}}$ & $2179^{\mathrm{B}}$ & $85^{\mathrm{C}}$ & $904^{\mathrm{A}}$ & $1473^{\mathrm{B}}$ & $0.46^{\mathrm{B}}$ & $0.72^{\mathrm{A}}$ & $0.72^{\mathrm{A}}$ \\
\hline Rayong 11 (G3) & $195^{\mathrm{D}}$ & $1273^{\mathrm{C}}$ & $2222^{\mathrm{B}}$ & $55^{\mathrm{D}}$ & $807^{\mathrm{B}}$ & $1371^{\mathrm{C}}$ & $0.40^{\mathrm{C}}$ & $0.65^{\mathrm{B}}$ & $0.66^{\mathrm{B}}$ \\
\hline CMR38-125-77 (G4) & $319^{\mathrm{A}}$ & $1449^{\mathrm{B}}$ & $2552^{\mathrm{A}}$ & $124^{\mathrm{A}}$ & $941^{\mathrm{A}}$ & $1600^{\mathrm{A}}$ & $0.48^{\mathrm{A}}$ & $0.70^{\mathrm{A}}$ & $0.67^{\mathrm{B}}$ \\
\hline 30 June 2015 (P1) & $271^{\mathrm{B}}$ & $1804^{\mathrm{B}}$ & $2297^{\mathrm{C}}$ & $91^{\mathrm{C}}$ & $861^{\mathrm{B}}$ & $1391^{\mathrm{B}}$ & $0.19^{\mathrm{C}}$ & $0.55^{\mathrm{D}}$ & $0.66^{\mathrm{B}}$ \\
\hline 5 October $2015(\mathrm{P} 2)$ & $287^{\mathrm{A}}$ & $1234^{\mathrm{C}}$ & $1740^{\mathrm{D}}$ & $144^{\mathrm{A}}$ & $834^{\mathrm{B}}$ & $1149^{\mathrm{C}}$ & $0.42^{\mathrm{B}}$ & $0.79^{\mathrm{A}}$ & $0.73^{\mathrm{A}}$ \\
\hline 20 May $2016(\mathrm{P} 3)$ & $213^{\mathrm{C}}$ & $1991^{\mathrm{A}}$ & $3027^{\mathrm{A}}$ & $37^{\mathrm{D}}$ & $1401^{\mathrm{A}}$ & $1750^{\mathrm{A}}$ & $0.77^{\mathrm{A}}$ & $0.75^{\mathrm{B}}$ & $0.59^{\mathrm{D}}$ \\
\hline 3 November 2016 (P4) & $284^{\mathrm{AB}}$ & $820^{\mathrm{D}}$ & $2389^{\mathrm{B}}$ & $103^{\text {B }}$ & $469^{\mathrm{C}}$ & $1417^{\mathrm{B}}$ & $0.43^{\mathrm{B}}$ & $0.63^{\mathrm{C}}$ & $0.63^{\mathrm{C}}$ \\
\hline $\mathrm{G} 1 \times \mathrm{P} 1$ & $343^{\mathrm{EF}}$ & $2046^{\mathrm{B}}$ & $2639^{\mathrm{E}}$ & $14^{\mathrm{I}}$ & $1309^{\mathrm{B}}$ & $1206^{\mathrm{GH}}$ & $0.06^{\mathrm{K}}$ & $0.67^{\mathrm{E}}$ & $0.47^{\mathrm{H}}$ \\
\hline $\mathrm{G} 1 \times \mathrm{P} 2$ & $1416^{\mathrm{B}}$ & $2539^{\mathrm{A}}$ & $2927^{\text {BC }}$ & $58^{\mathrm{G}}$ & $895^{\mathrm{DE}}$ & $1286^{\mathrm{EFG}}$ & $0.39^{\mathrm{H}}$ & $0.41^{\mathrm{H}}$ & $0.47^{\mathrm{H}}$ \\
\hline $\mathrm{G} 1 \times \mathrm{P} 3$ & $371^{\mathrm{E}}$ & $932^{\mathrm{I}}$ & $2805^{\mathrm{CD}}$ & $172^{\mathrm{C}}$ & $557^{\mathrm{H}}$ & $1540^{\mathrm{D}}$ & $0.58^{\mathrm{E}}$ & $0.67^{\mathrm{E}}$ & $0.57^{\mathrm{G}}$ \\
\hline $\mathrm{G} 1 \times \mathrm{P} 4$ & $1450^{\mathrm{B}}$ & $1448^{\mathrm{E}}$ & $1636^{\mathrm{I}}$ & $198^{\mathrm{A}}$ & $893^{\mathrm{DE}}$ & $1023^{J}$ & $0.81^{\mathrm{B}}$ & $0.84^{\mathrm{A}}$ & $0.70^{\mathrm{C}}$ \\
\hline $\mathrm{G} 2 \times \mathrm{P} 1$ & $266^{\mathrm{FGH}}$ & $1736^{\mathrm{C}}$ & $2694^{\mathrm{DE}}$ & $43^{\mathrm{H}}$ & $1234^{\mathrm{B}}$ & $1554^{\mathrm{CD}}$ & $0.20^{\mathrm{J}}$ & $0.76^{\mathrm{C}}$ & $0.60^{\mathrm{EF}}$ \\
\hline $\mathrm{G} 2 \times \mathrm{P} 2$ & $1400^{\mathrm{B}}$ & $1720^{\mathrm{CD}}$ & $2151^{\mathrm{FGH}}$ & $69^{\mathrm{G}}$ & $1026^{\mathrm{C}}$ & $1641^{\mathrm{C}}$ & $0.35^{\mathrm{H}}$ & $0.65^{\mathrm{E}}$ & $0.78^{\mathrm{A}}$ \\
\hline $\mathrm{G} 2 \times \mathrm{P} 3$ & $267^{\mathrm{FGH}}$ & $769^{\mathrm{J}}$ & $2188^{\mathrm{FG}}$ & $95^{\mathrm{F}}$ & $441^{\mathrm{I}}$ & $1559^{\mathrm{CD}}$ & $0.44^{\mathrm{G}}$ & $0.65^{\mathrm{E}}$ & $0.74^{\mathrm{B}}$ \\
\hline $\mathrm{G} 2 \times \mathrm{P} 4$ & $2068^{\mathrm{A}}$ & $1315^{\mathrm{F}}$ & $1682^{\mathrm{I}}$ & $134^{\mathrm{D}}$ & $915^{\mathrm{D}}$ & $1137^{\mathrm{HI}}$ & $0.85^{\mathrm{A}}$ & $0.83^{\mathrm{A}}$ & $0.73^{\mathrm{B}}$ \\
\hline $\mathrm{G} 3 \times \mathrm{P} 1$ & $222^{\mathrm{HI}}$ & $2110^{\mathrm{B}}$ & $2963^{\mathrm{B}}$ & $33^{\mathrm{H}}$ & $1501^{\mathrm{A}}$ & $1879^{\mathrm{B}}$ & $0.21^{\mathrm{J}}$ & $0.77^{\mathrm{BC}}$ & $0.65^{\mathrm{D}}$ \\
\hline $\mathrm{G} 3 \times \mathrm{P} 2$ & $1203^{\mathrm{C}}$ & $1336^{\mathrm{F}}$ & $2020^{\mathrm{H}}$ & $96^{\mathrm{F}}$ & $655^{\mathrm{GH}}$ & $1269^{\mathrm{FG}}$ & $0.48^{\mathrm{F}}$ & $0.55^{\mathrm{F}}$ & $0.68^{\mathrm{C}}$ \\
\hline $\mathrm{G} 3 \times \mathrm{P} 3$ & $174^{\mathrm{I}}$ & $605^{\mathrm{K}}$ & $2285^{\mathrm{F}}$ & $32^{\mathrm{H}}$ & $265^{\mathrm{J}}$ & $1255^{\mathrm{FG}}$ & $0.26^{\mathrm{I}}$ & $0.51^{\mathrm{G}}$ & $0.58^{\mathrm{FG}}$ \\
\hline $\mathrm{G} 3 \times \mathrm{P} 4$ & $2009^{\mathrm{A}}$ & $1040^{\mathrm{GH}}$ & $1618^{\mathrm{I}}$ & $57^{\mathrm{G}}$ & $807^{\mathrm{EF}}$ & $1081^{\mathrm{IJ}}$ & $0.67^{\mathrm{D}}$ & $0.76^{\mathrm{C}}$ & $0.74^{\mathrm{B}}$ \\
\hline $\mathrm{G} 4 \times \mathrm{P} 1$ & $252^{\mathrm{GHI}}$ & $2071^{\mathrm{B}}$ & $3813^{\mathrm{A}}$ & $58^{\mathrm{G}}$ & $1561^{\mathrm{A}}$ & $2363^{\mathrm{A}}$ & $0.29^{\mathrm{I}}$ & $0.79^{\mathrm{B}}$ & $0.62^{\mathrm{E}}$ \\
\hline $\mathrm{G} 4 \times \mathrm{P} 2$ & $2071^{\mathrm{A}}$ & $1620^{\mathrm{D}}$ & $2091^{\mathrm{GH}}$ & $140^{\mathrm{D}}$ & $868^{\mathrm{DE}}$ & $1370^{\mathrm{E}}$ & $0.45^{\mathrm{FG}}$ & $0.57^{\mathrm{F}}$ & $0.68^{\mathrm{C}}$ \\
\hline $\mathrm{G} 4 \times \mathrm{P} 3$ & $322^{\mathrm{EFG}}$ & $972^{\mathrm{HI}}$ & $2278^{\mathrm{F}}$ & $112^{\mathrm{E}}$ & $615^{\mathrm{H}}$ & $1314^{\mathrm{EF}}$ & $0.44^{\mathrm{G}}$ & $0.71^{\mathrm{D}}$ & $0.62^{\mathrm{E}}$ \\
\hline $\mathrm{G} 4 \times \mathrm{P} 4$ & $1037^{\mathrm{D}}$ & $1131^{\mathrm{G}}$ & $2026^{\mathrm{H}}$ & $185^{\text {B }}$ & $723^{\mathrm{FG}}$ & $1353^{\mathrm{EF}}$ & $0.74^{\mathrm{C}}$ & $0.73^{\mathrm{D}}$ & $0.75^{\mathrm{B}}$ \\
\hline
\end{tabular}

$\mathrm{CV}=$ Coefficient of variation, Values followed by the same letter are not significantly different by least significant difference test (at 0.01 probability level)

Table 5. Stepwise regression analysis for total dry weight, storage root dry weight at 270 days after planting (DAP) and other leaf characteristics of the four cassava genotypes

\begin{tabular}{|c|c|c|c|c|c|}
\hline Crop trait & Variable & Coefficient & $\mathbf{t}$ & $\begin{array}{c}\text { Determination coefficient } \\
\left(\mathbf{R}^{2}\right)\end{array}$ & P for regression \\
\hline \multirow[t]{6}{*}{ Total dry weight at 270 DAP } & Constant & 1634.04 & $4.88 * *$ & 0.6 & $<0.05$ \\
\hline & LAI at 180 DAP $\left(\mathrm{cm}^{2} \mathrm{~cm}^{-2}\right)$ & 195.92 & $3.97 * *$ & & \\
\hline & Leaf life during 90 DAP (days) & 8.86 & $2.72 * *$ & & \\
\hline & Leaf life during 180 DAP (days) & -6.15 & $-4.28 * *$ & & \\
\hline & Fallen leaf ratio for $90 \mathrm{DAP}$ & 6525.17 & $6.37 * *$ & & \\
\hline & Fallen leaf ratio for $180 \mathrm{DAP}$ & -1691.40 & $-4.47 * *$ & & \\
\hline \multirow[t]{5}{*}{ Storage root dry weight at $270 \mathrm{DAP}$} & Constant & 1179.15 & $5.48 * *$ & 0.5 & $<0.05$ \\
\hline & LAI at 180 DAP $\left(\mathrm{cm}^{2} \mathrm{~cm}^{-2}\right)$ & 71.67 & $2.25^{*}$ & & \\
\hline & LAI at 270 DAP $\left(\mathrm{cm}^{2} \mathrm{~cm}^{-2}\right)$ & -68.62 & $-3.33^{* * *}$ & & \\
\hline & Fallen leaf ratio for $90 \mathrm{DAP}$ & 4107.49 & $6.62 * *$ & & \\
\hline & Fallen leaf ratio for $180 \mathrm{DAP}$ & -702.30 & $-2.58^{*}$ & & \\
\hline
\end{tabular}

LAI $=$ Leaf area index, $\mathrm{t}=\mathrm{t}-$ (or student) test,$*=$ Significant difference at 0.05 probability level, $* *=$ Significant difference at 0.01 probability level 
CMR38-125-77 had the highest values of both total biomass and storage root dry weight at 270 DAP (Table 4) with high values of fallen leaf ratio (Table 3 ), but this genotype also produced more leaves during the growing seasons as indicated by high values of LAI (Table 3). Keating et al. (1982a) reported that shading effect and leaf abscission of cassava $M$ Aus 10 genotype grown at Queensland, Australia began at LAI values of 5 to 6 . Fallen leaf from senescence was normally observed in cassava and this also involves nutrient remobilization to stems and storage roots (Hortensteiner and Feller, 2002; Duque and Setter, 2013). Based on the association between fallen leaf ratio and dry weights (Table 5), further studies are still necessary to approve. Our results also demonstrated Rayong 9 genotype was the second in terms of LAI at 180 DAP and storage root dry weight at 270 DAP with showing the highest HI values at 180 and 270 DAP (Tables 3 and 4). The previous studies indicated that greater LAI increased cassava growth and yield (Cock et al., 1979; El-Sharkawy, 2003; Phuntupan and Banterng, 2017). Under the International Center for Tropical Agriculture (CIAT), Colombia condition, the optimal LAI for growth of storage root ranges from 3 to 3.5 and cassava crop should maintain this LAI level as long as possible to achieve high storage root yield (Cock et al., 1979). The relationship between high cassava crop growth rates and high leaf area indices of 10 or more in Queensland, Australia has been reported by Keating et al. (1982b). El-Sharkawy (2006) indicated that small leaf area of canopy (with the LAI values within 2) during 1-3 and 8-12 months after planting limited cassava storage root yield, indicating the important in breeding for greater and more sustainable canopy during most of the growth cycle with enhanced leaf photosynthesis, high harvest index and strong root sink. Our study pointed out that LAI at 180 DAP or during a stage of mid bulking and maximum canopy development (Alves, 2002) was a component crop trait associated with both final biomass and yield, and breeding to obtain high biomass and yield productivity with increasing this crop trait would also be an interesting issue for cassava breeders. The information from our study allows a valuable opportunity for designing ideal leaf performances to increase cassava yield (Jones et al., 2003). Cock et al. (1979) used a simulation approach to help for plant type design and they found that a combination of branching at about 30 weeks, leaf lives of 15 to 20 weeks, maximum leaf size of 500 to $600 \mathrm{~cm}$ and planting at 20,000 plants $\mathrm{ha}^{-1}$ under CIAT, Colombia with well crop management conditions presented the potential dry weight for cassava storage root yield of $30 \mathrm{Mg} \mathrm{ha}^{-1}$. A study of Lenis et al. (2006) under Colombia condition indicated positive relationship between cassava leaf retention based on visual score and storage root yield, and they suggested that this leaf trait could also be usefully incorporated into the concept of ideal plant type for cassava.

In addition, this research revealed that high total biomass and storage root dry weight at 270 DAP for the 20 May 2016 planting date (Table 4) also related with high value of LAI at mid bulking stage of cassava (Table
3), and this approves the potential of this leaf trait as a fundamental factor for designing a new plant type to increase cassava productivity. In order to improve cassava storage root yield based on the planting date management for this particular ecology and/or other similar environmental conditions, the results from this study also evinced that early rainy season planting dates would be more suitable option for cassava production than the late rainy season planting dates.

\section{CONCLUSION}

It could be concluded that higher temperature seemed to be related with longer leaf life during 180 DAP, large LAD values during 180-270 DAP, and greater values of LAI at 270 DAP. A combination of LAI at 180 DAP, leaf life during 90 and 180 DAP and fallen leaf ratio for 90 and 180 DAP was associated to total dry weight, and a combination of LAI at 180 and 270 DAP and fallen leaf ratio for 90 and 180 DAP contributed most to storage root yield. CMR38-125-77 genotype had high total biomass and storage root dry weight at 270 DAP with giving high LAI at 180 DAP. CMR38-125-77 was identified as a desirable genotype for storage root yield at the 30 June planting date. The 20 May 2016 planting date produced more total biomass and storage root dry weight than the other plating dates.

\section{ACKNOWLEDGEMENTS}

This study was supported by National Science and Technology Development Agency (NSTDA), Thailand. Assistance in conducting the work was also received from Agronomy Department and the Plant Breeding Research Center for Sustainable Agriculture, Khon Kaen University, Thailand.

\section{LITERATURE CITED}

Alves, A.A.C. 2002. Cassava Botany and Physiology. In: Cassava: Biology, Production and Utilization, ed. Hillocks, R.J., Thresh, J.M. and Bellotti, A.C., 67-89, CABI Publishing, New York, USA.

Analytical Software. 2013. Statistix 10. Analytical Software, Florida, USA.

An, D., J. Yong and P. Zhang. 2012. Transcriptome profiling of low temperature-treated cassava apical shoots showed dynamic responses of tropical plant to cold stress. BMC Genomics. 13: 64.

Anonymous. 2008. Good Agricultural Practices for Cassava. National Bureau of Agricultural Commodity and Food Standards Ministry of Agriculture and Cooperatives, Thailand.

Cock, J.H. 1984. Cassava. In: The Physiology of Tropical Field Crops, ed. Goldsworthy, P.R. and Fisher, N.M., 529-550, Wiley, Chichester, UK.

Cock, J.H., D. Franklin, G. Sandoval and P. Juri. 1979. The ideal cassava plant for maximum yield. Crop Sci. 19 (2): 271-279.

De Tafur, S.M., M.A. El-Sharkawy and F. Calle. 1997. Photosynthesis and yield performance of cassava in seasonally dry and semiarid environments. Photosynthetica. 33: $249-257$

Duque, L.O. and T.L. Setter. 2013. Cassava response to water deficit in deep pots: root and shoot growth, ABA, and carbohydrate reserves in stems, leaves and storage roots. Trop. Plant Biol. 6: 199-209. 
El-Sharkawy, M.A. 2003. Cassava biology and physiology. Plant Mol. Biol. 53: 621-641.

El-Sharkawy, M.A., A.D. Hernandez and C. Hershey. 1992. Yield stability of cassava during prolonged mid-season water stress. Exp. Agric. 28: 165-174.

Freed, R.D. and O. Nissen. 1992. MSTAT-C Version 1.42 Michigan State University, East Lansing, Michigan, USA

Gomez, K.A. and A.A. Gomez. 1984. Statistical Procedures for Agricultural Research. John Wiley and Sons, New York, USA.

Haggblade, S., A.A. Djurfeldt, D.B. Nyirenda, J.B. Lodin, L. Brimer, M. Chiona, L. Chiwona-Karltun, C. Cuambe, M. Dolislager, C. Donovan, K. Droppelmann, M. Jirström, E. Kambewa, P. Kambewa, N.M. Mahungu, J. Mkumbira, J. Mudema, H. Nielson, M. Nyembe, V.A. Salegua, A. Tomo and M. Weber. 2012. Cassava commercialization in Southeastern Africa. J. Agribus. Dev. Emerg. Econ. 2: 4-40.

Hortensteiner, S. and U. Feller. 2002. Nitrogen metabolism and remobilization during senescence. J. Exp. Bot. 53: 927-937.

Irikura, V., J.H. Cock and K. Kawano. 1979. The physiological basis of genotype-temperature interactions in cassava. Field Crops Res. 2: 227-239.

Jones, J.W., G. Hoogenboom, C.H. Porter, K.J. Boote, W.D. Batchelor, L.A. Hunt, P.W. Wilkens, U. Singh, A.J. Gijsman and J.T. Ritchie. 2003. The DSSAT cropping system model. Eur. J. Agron. 18: 235-265.

Kawano, K. 1990. Harvest index and evoluation of major food crop cultivars in the tropics. Euphytica. 46: 195-202.

Keating, B.A. and J.P. Evenson. 1979. Effect of soil temperature on sprouting and sprout elongation of stem cuttings of cassava (Manihot esculenta Crantz.). Field Crops Res. 2: 241-251.

Keating, B.A., J.P. Evenson and S. Fukai. 1982a. Environmental effects on growth and development of cassava (Manihot esculenta Crantz.) I. Crop development. Field Crops Res. 5: 271-281.

Keating, B.A., J.P. Evenson and S. Fukai. 1982b. Environmental effects on growth and development of cassava (Manihot esculenta Crantz) II. Crop growth rate and biomass yield. Field Crops Res. 5: 283-292.
Lahai, M.T., J.B. George and I.J. Ekanayake. 1999. Cassava (Manihot esculenta Crantz) growth indices, root yield and its components in upland and inland valley ecologies of Sierra Leone. J. Agron. Crop Sci. 182: 239-247.

Lebot, V. 2009. Tropical Root and Tuber Crops: Cassava, Sweet Potato, Yam and Aroids. CABI, UK.

Lemoine, R., S.L. Camera, R. Atanassova, F. Dédaldéchamp, T. Allario, N. Pourtau, J. -L. Bonnemain, M. Laloi, P. CoutosThévenot, L. Maurousset, M. Faucher, C. Girousse, P. Lemonnier, J. Parrilla and M. Durand. 2013. Source-to-sink transport of sugar and regulation by environmental factors. Front Plant Sci. 4: 1-21.

Lenis, J.I., F. Calle, G. Jaramillo, J.C. Perez, H. Ceballos and J.H. Cock. 2006. Leaf retention and cassava productivity. Field Crops Res. 95: 126-134.

Ogola, J.B.O. and C. Mathews. 2011. Adaptation of cassava (Manihot esculenta) to the dry environments of Limpopo, South Africa: growth, yield and yield components. Afr. J. Agric. Res. 6: 6082-6088.

Page, W.W., J.R.W. Harris and A. Youdeowei. 1990. Defoliation and consequent crop loss in cassava caused by the grasshopper Zonocerus variegatus (L.) (Orthoptera: Pyrgomorphidae) in southern Nigeria. Bull. Entomol. Res. 70(1): 151-163.

Phuntupan, K. and P. Banterng. 2017. Physiological determinants of storage root yield in three cassava genotypes under different nitrogen supply. J. Agr. Sci., Cambridge. 155: 978-992.

Roychowdhury, S. 1995. Leaf area development in colocasia and its relationship with yield. Indian J. Plant Physiol. 4: 305308.

Tan, S.L. and J.H. Cock. 1979. Branching habit as a yield determinant in cassava. Field Crops Res. 2: 281-289.

Thomas, H. and C.M. Smart. 1993. Crops that stay green. Ann. Appl. Biol. 123: 193-219. 